\title{
Advantages to Using Social-Behavioral Models of Medication Adherence in Research and Practice
}

\author{
K. Rivet Amico, $P h D^{7}$, M. Mugavero, MD², Marie A. Krousel-Wood, MD, MSPH ${ }^{3}$, \\ Hayden B. Bosworth, $P h D^{4}$, and Jessica S. Merlin, MD, MBA ${ }^{2}$
}

'University of Michigan, School of Public Health, Ann Arbor, MI, USA; ${ }^{2}$ Division of Infectious Diseases, Department of Medicine, University of Alabama at Birmingham, Birmingham, AL, USA; ${ }^{3}$ Tulane University Schools of Medicine and Public Health and Tropical Medicine and Ochsner Health System, New Orleans, LA, USA; ${ }^{4}$ Center for Health Services Research in Primary Care, Durham VAMC, Duke University Medical Center, Durham, NC, USA.

Achieving and sustaining high levels of adherence to medication regimens is essential to improving health outcomes, but continues to be a challenge for a sizable proportion of patients. Decades of research suggests that medication adherence is determined by a complex constellation of factors. Social-behavioral science research has focused on creating frameworks that identify which contextual, personal, social, or drug-related factors appear to most influence adherence. Comprehensive models of adherence propose specific structural relationships between these factors that can be used to plan for, implement, and monitor programs that seek to optimize adherence. The use of social-behavioral models offers multiple advantages in both practice and research environments; however, the breadth and depth of these models can deter many from engaging in this important exercise. To promote the use of social-behavioral frameworks and models of adherence, we provide a brief overview of the advantages in using a social-behavioral lens in adherence work, a sampling of models used in HIV medication adherence research that have high generalizability to other conditions, and practical guidance for grounding adherence promotion strategies in evidence informed by socialbehavioral science research.

KEY WORDS: social-behavioral models; health behavior; adherence models; medication adherence; HIV.

J Gen Intern Med 33(2):207-15

DOI: $10.1007 / \mathrm{s} 11606-017-4197-5$

(c) Society of General Internal Medicine 2017

\section{INTRODUCTION}

Long-term medication adherence is a well-recognized requirement for positive health outcomes for many chronic diseases. ${ }^{1}$ While medication side effects, toxicities, and burdens of treatment for chronic diseases have improved dramatically in recent decades, patients often struggle to achieve adequate medication adherence rates. In the US alone, costs related to poor medication adherence are in the billions of dollars each year. $^{2,3}$ Not surprisingly, efforts to better understand the determinants and facilitators of, and challenges to, adherence have proliferated in medicine and social science, contributing to a robust body of literature that can seem overwhelming. ${ }^{4}$

Published online December 4, 2017
In response to the ongoing need to optimize work with patient medication adherence in a manner that leverages social-behavioral science, the University of Alabama at Birmingham (UAB) hosted a conference, "Understanding and Improving Treatment Adherence: An Interdisciplinary Approach," in February 2016. Our review and discussion of social-behavioral models of medication adherence provides a rationale for their wide-scale use, a brief sampling of HIV medication adherence models that can be generalized to other conditions and regimens, a synthesis of salient factors identified across multiple models, and practical recommendations for using evidence-based and evidence-informed adherence models to guide practice and research. Here we summarize this portion of the working group meeting to bridge the gap between social-behavioral science and the diverse disciplines engaged in providing high-quality patient care. The objective of this review is to build confidence in the utility of fully leveraging social-behavioral theories, models, and frameworks in both clinical work and research.

\section{Adherence to Medication Regimens Is Often Far from} "Simple". To date, a number of "reasons" for non-adherence have been well-documented, ranging from "just forgetting" to complex social negotiations of avoiding dosing to avoid disease-related stigma. ${ }^{5,6}$ From a social-science perspective, any reason has underlying causal mechanisms (e.g., forgetting resulting from lacking a skill set to dose in a timely manner) that relate to factors important in most decision-making and behavior change efforts (e.g., attitudes and beliefs - the lack of skills may be driven by beliefs that daily dosing is not really needed). Rather than assuming that adherence results from being advised to adhere by a medical provider, or knowing that non-adherence will be unhealthy in the short or long term, a social-behavioral perceptive identifies multiple underlying causes of medication adherence (and suboptimal adherence). Theoretical principles that come from diverse areas of study, including the fields of cognition, behavior change, motivation, coping, affect regulation, and decision making, provide a strong basis for the assertion that self-administration of medication regimens is a complex phenomenon. Like most human behavior, dosing behavior happens in a larger context of individual beliefs, social experiences, anticipated outcomes, and myriad "ways of being" that include culture perspectives and worldviews. While the act of self-administration of a 
medication may appear discrete and "simple," the models of adherence we consider below suggest that this observable behavior has dynamic, but identifiable, sets of precursors.

\section{Adherence from a Social-Behavioral Perspective?}

One benefit of applying social-behavioral models is that such models offer a comprehensive mosaic of factors related to optimal and suboptimal adherence based on evidence accumulated through research on "real-world" experiences with medication adherence, each factor offering a potential path for intervention. Many providers have experienced the ineffectiveness of focusing on a single factor influencing adherence (e.g., information only - simply telling patients to take their medications as prescribed, and that not doing so will lead to complications). Appreciating other factors found to influence adherence, directly or indirectly, provides a greater opportunity to help patients optimize their adherence. The space between having medications in hand and benefiting from them is intersected by multiple social-behavioral facilitating and challenging pathways. Given the growing call for patient-centered and differentiated care, adopting and sharing complex perspectives of patient adherence that help to gain an optimal understanding of patient challenges and experiences, and that avoid centering on patient performance (e.g., good patients adhere and bad patients do not) can be facilitated through adoption of comprehensive conceptualizations of adherence outcomes.

HIV provides an example of the benefit of considering a comprehensive view of factors related to optimal and suboptimal adherence. In the case of HIV treatment, achieving and sustaining suppression of the virus (i.e., a desired clinical outcome) requires adequate adherence to antiretroviral therapy (ART). Shortly after the wide-scale introduction of ART in the US in 1996, structural and medication-related factors were identified as important barriers; however, many other important factors emerged over time, including acceptance of the disease, negotiation of stigma, perception of the regimen as needed/helpful, and positive attitudes towards the regimen and regimen effects. Social-behavioral models detailing underlying drivers of HIV medication adherence provided a framework from which practitioners and researchers could identify and work with the complexities of ART adherence, and support patients in achieving positive clinical outcomes.

A second important contribution of social-behavioral models of medication adherence centers on providing a clear architecture for intervention implementation and effect monitoring. In an environment of limited resources for practice and research efforts, having a clear, evidence-informed model for why an intervention program or strategy would be expected to have a positive effect is essential. Social-behavioral models identify the causal factors that must change for an intervention to be effective. Change in theory-identified factors (e.g., selfefficacy, skills in managing side effects) as a result of a new service or intervention provides explanations for why an effect is achieved. Alternatively, failure to see changes in factors underlying medication adherence may explain where an intervention or service failed. Simply stated, focusing on just the primary endpoint (i.e., adherence) without unpacking the drivers of intervention effects fails to fully explain supportive or null findings.

\section{Social-Behavioral Models of Medication Adherence (Examples from HIV Medication Adherence)}

Specific to HIV medication adherence, there are a number of social-behavioral models that have been evaluated, used for intervention design, and adapted over time. From these models, core "drivers" of medication adherence can be identified, and likely generalized to medication adherence for other conditions.

Munro and colleagues provided a comprehensive overview of models in the evidence base through $2006 .^{7}$ These include established health behavior models, such as the Health Belief Model $^{8,9}$ and Information, Motivation, Behavioral Skills (IMB) models, ${ }^{10-12}$ as well as Social Cognitive Theory (SCT), ${ }^{13,14}$ the Theory of Reasoned Action ${ }^{15,}{ }^{16}$ and Planned Behavior, ${ }^{17,}{ }^{18}$ Protective Motivation Theory, ${ }^{19}$ and the Transtheoretical Model (TTM). ${ }^{20,21}$ While all approaches offer a benefit to understanding ART adherence, no single model has emerged as superior.

Across these models, there are several common components. Core constructs shared across explanatory adherence models include a level of accurate information about or knowledge of the disease; the role of treatment and the specifics about following the treatment recommended; awareness or some sense of need for the treatment; motivation to adhere (such as attitudes and beliefs about adherence and non-adherence, with different models detailing various core drivers of motivation); and a skill set needed to execute adherence behavior across diverse situations and settings. Models differ in how community- and structural-level factors are incorporated. Many models include such factors as relevant to the extent that they are incorporated into individual beliefs and experiences, while others characterize systematic levels of influence as contributing independently to adherence, as the environment in which care is negotiated sets the context for any individuallevel experiences with care.

Table 1 presents factors that are considered critical across several models, as well as the constellation of factors needed to establish and sustain optimal levels of medication adherence according to each theory. Readers are encouraged to use the references and resources provided to further explore the details and literature pertaining to specific models of interest. Most of the models and frameworks in Table 1 are explanatory or predictive in nature, focusing on causes of medication adherence, but typically do not specify how to change adherence behavior (except 
Table 1 Extrapolated Core Constructs and Predictions of Leading Models of Adherence

\begin{tabular}{|c|c|c|}
\hline Model/Theory & Constructs & Who will adhere? \\
\hline
\end{tabular}

Information Motivation Behavioral Skills (IMB) model of adherence ${ }^{23}$

Situated IMB model of Care Initiation and Maintenance $(\text { sIMB-CIM) })^{24}$ Social Cognitive Theory
(Cognitive Theory)

Self-Regulation Theory ${ }^{25,} 26$

${ }_{18}^{\text {Theory of Planned Behavior }}{ }^{17}$
Modifying variables are identified.

- Information about the regimen (when to take doses, special instructions, purpose of regimen), low misinformation (inaccurate beliefs about medications that are believed to be true) about regimen or adherence - Personal motivation (beliefs and attitudes about the consequences of adherence and non-adherence)

- Social norms (beliefs about norms for medication-taking and influence of significant others on adherence that facilitate adherence)

- Skills to execute adherence (having the skills to dose on time and as directed with the self-efficacy to do so). Skills directly impact adherence (e.g., it is not sufficient to be well-informed or motivated; skills are essential for translating information and motivation to action).

Modifying variables (acute depression, active substance use) are identified as moderators of information, motivation, and behavioral skills.

- An application of the IMB model to engagement in chronic care that identifies specific aspects of information, motivation, and behavioral skills nested within the social, cultural, and structural environment in which care is negotiated

- Information about care, costs, demands of self-care, and benefits that are facilitative (and low misinformation in selfand community-creating conflicts for uptake of care)

- Personal and social motivation (attitudes, beliefs, and emotions regarding condition and care, and the need, value, and costs of engagement in care to self and important others)

- Skills to engage in care in contexts common in cohort or community, access available care, garner needed support, use local resources, manage care with competing priorities/demands (child care, unemployment, substance use), and manage affect/stigma

Health behavior is a function of the

- Environment (stimuli from surrounding environment and reinforcement to adopt or avoid adherence)

- Person (attitudes, beliefs, expectations about outcomes, previous experiences, self-efficacy, motivation)

- Behavioral factors are identified, including self-observation, judgment, and reaction, that contribute to adherence.

Multicomponent process where behavior is a function of

- Illness experiences (such as experience of side effects or symptom reductions)

- Social interactions, information/knowledge, and affect related to illness and medications

- Cognitive processes that evaluate and adjust behaviors based on experiences

Illness and medication representations (the net result of experiences, affect towards, and ongoing evaluation of past and new experiences) are critical to self-regulation.

- Intentions to act (willingness and commitment to adhere)

- Attitudes (beliefs about adherence)

- Subjective norms (social pressure to adhere or not and relevance of that pressure)

- Perceived behavioral control (perceived ability and self-efficacy to adhere)

Specifically, beliefs and attitudes impact intentions, and intentions are also influenced by norms and social influence, as well as control beliefs. Intentions and behavioral control impact behavior.
Those who are well-informed about adherence and feel positively towards it, and who have social support for it, are more likely to have skills for adherence and confidence in executing those skills that determine adherence.

Those with facilitative knowledge about regimen and care, beliefs, attitudes, and feelings about care, and skills to manage care in the situations most common in a given community or cohort (facilitated by environments where community- and structurallevel factors contribute to positive beliefs and experiences with adherence and engagement in care)

Those with greater cues and supports in their environment and higher positive expectations of being able to reap the benefits of ART and realized benefits and skills

Those with expectance of positive outcomes from medication-taking, skills (and supporting experiences) in executing medication-taking, and affective (mood) processes that facilitate adherence ${ }^{26}$

Those with high intentions (commitment) towards adherence who have positive beliefs about adherence in terms of outcomes, receive social pressure to adhere from people who are important, and have a sense of being able to execute the needed behaviors and skills to adhere the IMB model, ${ }^{23}$ which does specify a process for intervention development). Thus these models articulate the factors that should be optimized or reduced, while the specifics on how to do that requires additional steps. 
Table 1. (continued)

\begin{tabular}{|c|c|c|}
\hline Model/Theory & Constructs & Who will adhere? \\
\hline Protective Motivation Theory ${ }^{19}$ & $\begin{array}{l}\text { Behavior (adherence) is caused by } \\
\text { - Sufficient motivation to protect oneself from negative } \\
\text { outcome } \\
\text { - Protective motivation as a function of appraisals of threat } \\
\text { and coping } \\
\text { - Threat appraisal as a function of perceived severity and } \\
\text { vulnerability to negative outcome relative to the intrinsic } \\
\text { and extrinsic rewards attributed to adherence or } \\
\text { non-adherence } \\
\text { - Coping appraisal as a function of perceived efficacy of a }\end{array}$ & $\begin{array}{l}\text { Those with higher perceived rewards of adherence } \\
\text { and higher perceived susceptibility to ill effects of } \\
\text { non-adherence and beliefs in ill effects being severe } \\
\text { will have a high threat appraisal, which, combined } \\
\text { with coping appraisal favoring adherence (beliefs } \\
\text { that adherence will bring positive outcomes as well } \\
\text { as having confidence in being able to implement } \\
\text { adherence behaviors that outweigh the perceived } \\
\text { costs of adherence), have high protective motivation } \\
\text { that will produce adherence. }\end{array}$ \\
\hline
\end{tabular}

Transtheoretical Model $^{20,21} \quad \begin{aligned} & \text { to the overall costs of adherence } \\ & \text { - Maps stages of "readiness" to adopt, enact, or maintain }\end{aligned}$ - Maps stages of "readinesence, which include

- Precontemplation (no recognition of need for adherence)

- Contemplation (thinking about need for adherence or improving it)

- Preparation (planning to adhere or improve)

- Action (implementing adherence or new behaviors to improve it)

- Maintenance (ongoing adherence and use of strategies to be adherent)

Chronic Care $\operatorname{Model}^{27,} 28$

Core factors influencing health behavior include

- Self-management (see below)

- Delivery of care systems

- Decision making

- Clinical information systems

- Community (resources and policies)

- Health systems in their organization of health care

delivery

These actors produce informed and activated patients and prepared and proactive practice teams that further interact.

Self-Management Theory ${ }^{29}$ Management of a self-administered behavior (adherence) is a distal outcome that is a function of

- Context (risk and protective factors including access to care and culture)

- Process (knowledge and beliefs, self-efficacy, skills)

- Abilities (goal-setting, self-monitoring, reflective thinking, planning, emotional control)

- Social facilitation (influence, support)

- Proximal outcomes (self-management behaviors and associated real and perceived costs of adherence)

Critical determinants of clinical outcomes are a function of

- Predisposing characteristics of patient (age, sex, race/ethnicity, insurance)

- Enabling characteristics (attitudes, knowledge, preferences)

- Need characteristics (comorbidities)

- Environmental characteristics (distance to travel to care or get medications, rural/urban)

- Provider/care characteristics (wait times, quality care)

Applied to ART adherence as external environment (life activities), health care environment (system, clinical, and provider factors) and patient predisposing (stigma, substance abuse), enabling (social support, housing, insurance), and perceived need (health beliefs, symptoms), producing adherence and retention in care.

The patient and his/her behavior are the result of

- Individual level (risk perception, self-efficacy, motivation)

- Micro level (interactions with close significant others)

- Meso level (community-level factors including stigma,

distance to care, quality of care)

- Macro level (drug supply, health systems, law and policy)
Individuals in action and maintenance are adherent. Note that motivation as a global construct is the driving force behind movement between stages.

Those who are informed and activated (engaged, empowered, and invested in self-care) due to wellcoordinated and responsive medical care systems that promote and facilitate (through tools and access) ongoing self-management of disease

Those who execute self-management behaviors have facilitating contexts (environmental support, cultural support, and access to quality care), and have positive and strong process abilities (are wellinformed, feel capable of managing their own behaviors and can adapt to emerging needs/demands, and have strong social facilitators). Positive experiences with outcomes and improvements in quality of life reinforce the process.

Those with facilitating predisposing, enabling, and needs characteristics, in the context of facilitating environmental and care/provide characteristics

Those who have facilitating factors in each of the levels (immediate social environment, community and local care level, and systems of care and policies level) have higher accurate risk evaluation, efficacy, and motivation to adhere.

$A R T$, antiretroviral therapy; IMB, Information, Motivation, Behavioral Skills

Note: Communication and counseling models (e.g., motivational interviewing, patient-centered care, next-step counseling, problem-solving therapy) are not included but can be considered for how to intervene with the factors identified as critical to adherence

\section{Practical Guidance for Working with a Social-Behavioral Model of Adherence}

Optimizing research and practice through the use of a socialbehavioral model to ground interventions involves (1) selecting a model, (2) tailoring the model, (3) operationalizing factors in the model, and (4) actually putting the model and model-based interventions or programs into action by using it. Each step is summarized below in terms of considerations and action items. 
We also provide an example of each step in Table 2, for the development, implementation, and evaluation of the iENGAGE intervention targeting viral suppression among people living with HIV newly entering clinical care, based on a situated IMB model.

Table 2 Application of Recommended Practical Guidance for Working with a Social-Behavioral Model of Adherence: the Situated IMB Theory-Based Integrating ENGagement and Adherence Goals Upon Entry (iENGAGE) Behavioral Intervention Consisting of Four In-Person Counseling Sessions Over 12 Months Among Persons Newly Initiating Outpatient HIV Medical Care at Four HIV Clinics in the United States (5R01AI103661-05)

\begin{tabular}{ll}
\hline \hline Steps & Application for iENGAGE \\
\hline 1. Select a model & $\begin{array}{l}\text { - Situated Information Motivation Behavioral Skills (sIMB), } \\
\text { addressing distinct, but interrelated behaviors of adherence to }\end{array}$ \\
& $\begin{array}{l}\text { HIV medical appointments (adherence to care) and adherence } \\
\text { to antiretroviral therapy (adherence to medications) }\end{array}$
\end{tabular}

2. Tailor the model

3. Operationalize factors

4. Use it
- Engagement of persons living with HIV, case managers, behavioral scientists, and medical providers to identify unique considerations to be accounted for and emphasized with implementation of the sIMB model for iENGAGE

- Emphasis placed on situating adherence related content to the range of psychological, social, and cultural factors and experiences of persons newly diagnosed with HIV initiating medical care

- Adaptation to address the distinct and shared information, motivation, and behavioral skills for the adherence to care and adherence to medications behaviors

- Tailoring to focus SIMB-guided intervention content upon proactive development and maintenance of effective HIV self-care adherence behaviors, rather than reactively improving behaviors among individuals demonstrating challenges with adherence

- Engagement of persons living with HIV, behavioral scientists, and medical providers to identify specific factors most relevant to adherence behaviors among persons newly initiating HIV medical care

- Theory-guided content on information, motivation, and behavioral skills specifically developed for persons with no/limited prior exposure and experience with HIV, as well as no/limited experience navigating the health care system and engaging in self-care adherence behaviors for other medical conditions

- A range of structural (e.g., transportation, health insurance, housing) and psychosocial (e.g., disclosure, stigma, self-efficacy) factors identified as relevant to acquisition of information, motivation, and behavioral skills, and included in an adapted adherence screener

- Identification of validated questionnaires to assess HIV information, motivation, and behavioral skills for adherence to care and for adherence to ART, aligned with sIMB constructs - Intervention mapping procedures followed for implementation of SIMB theory-guided iENGAGE behavioral intervention at 4 study sites

- Each iENGAGE session generally broke into: rapport-building (first $5 \mathrm{~min}$ ), followed by providing, clarifying, and discussing relevant information (15 min), and the remainder of the visit engaging in motivational interviewing-informed exploration and problem-solving around use of HIV care and ART medications (10-45 min) - An adapted adherence screener was used to identify potential barriers to returning for their next clinic medical visit and barriers to ART adherence to guide delivered intervention content including information, motivation, and behavioral skills to address barriers

- Enrollment of 372 iENGAGE participants across 4 study sites, including 186 participants assigned to intervention arm, with intervention delivery complete, closure of study database, and evaluation of primary and secondary study outcomes in process

\section{Practical considerations}

- IMB widely used in HIV adherence and behavior change research

- "Situated" aspect emphasizes the role of social, cultural, structural, and economic influences germane to adherence in the broader context of care and ART

- Substantial knowledge and application of IMB and sIMB theory-based intervention development, implementation, and evaluation among study team

- Persons newly initiating HIV medical care commonly report feeling "overwhelmed," both psychologically and with the medical information provided

- Intervention components, manual and counselor training, with emphasis on awareness and communication "situated" to the psychological distress expressed by participants

- Session 1 content more structured and focused upon general information about HIV infection and the role of adherence to care and ART medications, with focus on participant-identified motivation to attend next scheduled medical appointment - Content of sessions 2-4 more flexible; aligned with sIMB constructs and tailored to the specific and most pressing needs of each participant, focusing upon each participant's accumulated knowledge (information) and experiences with motivation and behavioral skills to address the most pressing adherence behavior

- Limited published data among persons newly initiating HIV medical care to inform process

- Leveraged substantial experience operationalizing IMB and sIMB theory- based intervention among iENGAGE behavioral scientists

- Leveraged experience of clinical programs (e.g., new patient orientation/navigation), providers, and persons living with HIV at participating study sites to operationalize factors relevant to iENGAGE study population

- Engagement of clinical and behavioral research content experts beyond the immediate iENGAGE study team to assist in process of operationalizing factors

-2-day interventionist training prior to trial enrollment included sIMB model constructs, motivational interviewing skills, and strategies

- 1-day booster interventionist training at the end of year 1

- Weekly interventionist calls with iENGAGE behavioral

scientists to discuss experiences delivering content in context of theoretical underpinnings of intervention

- Qualitative interviews with interventionists at conclusion of study to ascertain experiences delivering content and to assess alignment/fidelity with sIMB theoretical underpinnings - Systematic evaluation of the effects of the iENGAGE intervention (vs. standard--of-care control) on the acquisition of information, motivation, and behavioral skills for adherence to care and adherence to ART

- Systematic evaluation of the effects of the iENGAGE intervention (vs. standard-of-care control) on measured adherence to care and adherence to ART

- Mediation analysis planned to evaluate whether intervention effects on adherence to care and/or adherence to ART are attributable to differential adherence information, motivation, and behavioral skills acquisition in the intervention vs. control arms 
Note that there are a number of strategies to move from model to intervention programs, and the steps provided here are meant to highlight the general process of using social-behavioral models.

\section{SELECTING A MODEL}

From the models of HIV medication adherence, one or a combination of models often resonates with a service provider's or researcher's experiences in working with ART or other medication adherence. Reviews of models have been conducted in an attempt to establish empirical superiority of one model over others. A recent meta-analysis ${ }^{34}$ reviewing 124 theory-based adherence interventions across diseases found that motivational interviewing ${ }^{35}$ (more a communication approach that also identifies sources for behavior change $e^{36}$ than a theory or model per se) was the most commonly used, followed by SCT, and then the TTM, self-regulation, cognitive theory, IMB, and self-management. Common use, however, does not speak to efficacy of a given model in relation to others or in relation to no model at all.

There is support for adopting a model to guide intervention development versus not using a model. For example, a review of 85 internet-based health behavior interventions engaging over 43,000 participants suggested that better intervention effects were achieved through reliance on a health behavior model to guide intervention components than with no model. ${ }^{37}$ Meta-analyses of interventions are somewhat limited in their ability to sort out effects of model-based versus atheoretical adherence intervention approaches because of heterogeneity. This heterogeneity may underlie failures to identify a significant advantage of interventions with an articulated theoretical basis as reported in some reviews. ${ }^{38,}{ }^{39}$ Empirical support for each model included in Table 1 is readily available in the literature. Once a model is selected, additional considerations are needed before the model can offer full utility in a given application; these consideration include tailoring the model to a specific context, operationalizing core factors, and using the model.

\section{TAILORING THE MODEL}

Models identify the core factors and pathways that facilitate or deter the adoption of the behavior needed to achieve the health outcome, but they often lack specific details about what the factor really looks like for a specific group or population. For example, the IMB model specifies that medication adherencerelated information is a critical component in medication adherence. However, additional elicitation work within a given cohort or community is needed to identify the specific kinds of information that promote adherence and the kinds of misinformation that impede medication adherence. Similarly, in several models, negative adherence-related attitudes are identified as problematic, as they create motivation not to adhere. To intervene, one must first identify the specific attitudes or beliefs that are posing the greatest problems in a given sample, cohort, or patient. Models identify highly generalizable constructs, such as awareness, motivation, or self-regard, and propose the structural relationships between these factors (what leads to what, what mediates which relationships); intervention strategies try to optimize adherence by impacting these broad factors in positive ways. Once you have your model of which factors matter the most at a general level, you next need to get very specific. We recommend using models to focus efforts to get specific (see below) and evaluate, propose, or offer tailored and targeted, theory-informed intervention strategies.

\section{OPERATIONALIZING FACTORS}

Whereas a model or theory identifies areas that are important in medication adherence, how each factor operates, exists, or is operationalized in a given cohort can be articulated a number of ways, including basic elicitation work and the use of process planning models. Elicitation work can be thought of as a qualitative and quantitative evaluation of the theory-related needs of a given patient, patient group, or larger group. The IMB model of adherence, ${ }^{23}$ for example, suggests that effective interventions require focus groups or interviews to identify which kinds of information, motivation, and skills are most pertinent to the target population or person. An application of the IMB model to initiation and retention in care for chronic diseases further emphasizes the critical role of this process, noting that all core constructs in the model must be situated to a specific group of patients or community for the model and model-based interventions to have utility (a situated IMB model [sIMB]). ${ }^{24}$ While constructs in socialbehavioral models help to narrow the scope of all possible facilitators and barriers to those that are identified by the model as most critical, it is possible to use current literature, patient and stakeholder groups, surveys, and interviews with current patients to articulate the specific needs in a given community in each area.

\section{USING IT}

Once a model is selected and the model-based factors operationalized in reference to a specific disease, regimen, or population, and the core general constructs have been operationalized, the model should be evaluated for utility by using it in practice and research. Several process approaches can inform the use of a model in quality improvement efforts. The plan, do, check, act (PDCA) ${ }^{40}$ the PRECEDE-PROCEDE model, ${ }^{41}$ the six Steps for Quality Intervention Development (6SQuID), ${ }^{42}$ and Intervention Mapping ${ }^{43}$ all use models in their approaches to intervention development, implementation, and evaluation. Readers are encouraged to consult these strategic approaches in the development of intervention strategies. However, even in the absence of these evaluation 
methods, any clinic or program seeking to enhance clientcentered adherence care can use an adherence model to identify potential model-based gaps, challenges, and resources of relevance in patient populations.

A model-based, well-articulated understanding of relevant and modifiable constructs in the pathway of factors influencing medication adherence can guide evaluation of programs and the construction of new ones. At the most basic level, supports for adherence and related tools or strategies should address one or more of the model-based factors. The need to address model-based factors is of particular importance in the case of interventional research, where model-based interventions can be evaluated for effects on adherence and the modelbased factors that lead to adherence (e.g., does participation in an intervention increase motivation, shift attitudes, or enhance skills?). The use of models in clinical care is also useful.

As an example of the utility of using models in clinical care, clinics may provide patients with pillboxes to facilitate adherence. From an IMB model perspective, providing pillboxes to patients would be expected to assist patients who have deficits in organizational and memory skills for dosing. The model suggests that by reducing the difficulty of dosing, one would need fewer behavioral skills to be able to dose successfully. The easier it is to dose, the lower the demands on motivation will be. The more complex a regimen, the more well-informed and highly motivated one needs to be to bring multiple skills to bear for successful adherence. Whether a pillbox would be expected to help depends on where the deficits causing non-adherence are most acute. If pillboxes are provided without attention to other critical determinants of adherence, their use may have a minimal or even negative impact on adherence.

The IMB model identifies motivation as a critical factor in adherence. In the case of patients struggling to develop positive beliefs about the effects of a regimen, reducing the difficulty of dosing through organizational tools is not the issue. In the case of patients who have a system of dosing in place that works well for them (e.g., has a bottle that is kept in view as a cue) but have deficits in other areas, introducing a pillbox misses the mark, and may actually further erode adherence by perturbing a dosing strategy that would work fine if other drivers of adherence were in place (e.g., high levels of knowledge and positive motivation).

The point of the prior discussion is that client-centered care relies on identification of specific deficits in factors that drive adherence. Identifying which relevant gaps are causing problems is facilitated by exploration of model-based factors (in this case, the kinds of information, attitudes, and beliefs about the personal and social consequences of adherence and nonadherence, and pertinent skills needed to execute dosing in commonly occurring situations). Rather than eliciting every possible reason for a missed dose, model-based discourse focuses on relative strengths and challenges in the social, contextual, and personal factors identified as requisite for dosing. Asking about model-based factors can make discourse more efficient and targeted.

Using a model to guide research and practice offers opportunities to better appreciate the complexities of adherence, engage in exploration of gaps and resources in a more efficient manner, and identify adherence promotion strategies that bridge specific gaps in model-based factors, and ultimately can be evaluated formally and informally to determine whether the model-based factor and adherence outcomes change as expected. Moreover, patient-centered care may be positively impacted by adopting an approach to patient discourse that highlights the importance of the myriad factors that contribute to optimal, long-term patient adherence.

\section{CONCLUSIONS}

Social-behavioral models can assist research and practice by consolidating the multitude of "reasons" for non-adherence into superordinate factors that drive adherence. These factors can then be operationalized and serve as the focus of intervention efforts and patient-provider discourse. We have provided a sampling of adherence models for readers to consider, as well as a series of steps that can be used in "actioning" a model, from a description of adherence in general to a powerful tool in understanding, intervening in, and monitoring adherence for specific conditions and cohorts. There is no consensus on a single "most effective" model, although many models share common core factors (e.g., motivation and skills).

Although models have high generalizability in their constructs, the steps we provide here for using a model highlight the need to bring broad constructs (e.g., motivation) down to the level of intervention target (e.g., the kinds of beliefs most impactful to a given cohort). Tailoring models may result in limiting their generalizability across cohorts. However, with client-centered interventions, one would expect to have highly generalizable constructs (e.g., regimen knowledge), with highly specific tailoring used to identify deficits and promote optimization (e.g., knowledge of dosing requirements for insulin-dependent diabetes). Further, if research were to develop higher standards for reporting on the factors that an intervention targeted to improve adherence and whether improvements were achieved on those factors, some strategies developed for one condition, regimen, or population could be considered and evaluated in other programs, using similar models but with different conditions, regimens, or populations.

There is growing consensus on the value of using wellarticulated models to conduct and evaluate research-as the measurement of changes in factors targeted by an intervention can helped to unpack intervention effects. The use of an adherence model to provide clear, evidence-informed formulation of how the core factors of the model operate within 
specific communities can facilitate a systematic evolution of intervention programs that effectively support medication adherence and other health behaviors.

Acknowledgements: Dr. Bosworth was supported in part by a VA Health Services Research and Development Senior Career Scientist award (VA HSRD 08-027). Dr. Merlin is supported by Agency for Healthcare Research and Quality grant K23MH104073. The authors would like to thank the UAB "Understanding and Improving Treatment Adherence: An Interdisciplinary Approach" conference leaders, as well as Elizabeth Rahn, $\mathrm{PhD}$, for their review and input in the preparation of this manuscript, and Stacey C. Tobin, PhD, for providing editorial support.

Corresponding Author: K. Rivet Amico, $P h D$; University of Michigan, School of Public Health, Ann Arbor, MI, USA (e-mail: ramico@umich. edu).

\section{Compliance with Ethical Standards:}

Conflict of Interest: Dr. Amico has received an education grant from Gilead Sciences through the University of Michigan. Dr. Bosworth has received consulting funds from Genentech, Sanofi, and Boehringer Ingelheim. Dr. Bosworth has also received grant funding from Sanofi, Johnson \&.Johnson, Takeda, and Improved Patient Outcomes. All other authors declare that they have no conflict of interest.

\section{REFERENCES}

1. Krueger KP, Berger BA, Felkey B. Medication adherence and persistence: a comprehensive review. Advances in therapy. 2005;22(4):313356.

2. Osterberg L, Blaschke T. Adherence to medication. The New England journal of medicine. 2005;353(5):487-497.

3. Sokol MC, McGuigan KA, Verbrugge RR, Epstein RS. Impact of medication adherence on hospitalization risk and healthcare cost. Medical care. 2005;43(6):521-530.

4. Zullig LL, Granger BB, Bosworth HB. A renewed Medication Adherence Alliance call to action: harnessing momentum to address medication nonadherence in the United States. Patient preference and adherence. 2016; 10:1189-1195.

5. Brown MT, Bussell JK. Medication adherence: WHO cares? Mayo Clinic proceedings. 2011;86(4):304-314.

6. AlGhurair SA, Hughes CA, Simpson SH, Guirguis LM. A systematic review of patient self-reported barriers of adherence to antihypertensive medications using the world health organization multidimensional adherence model. Journal of clinical hypertension (Greenwich, Conn.). 2012;14(12):877-886.

7. Munro S, Lewin S, Swart T, Volmink J. A review of health behaviour theories: how useful are these for developing interventions to promote long-term medication adherence for TB and HIV/AIDS? BMC public health. 2007;7:104.

8. Becker MH, Radius SM, Rosenstock IM, Drachman RH, Schuberth KC, Teets KC. Compliance with a medical regimen for asthma: a test of the health belief model. Public health reports (Washington, D.C. : 1974). 1978;93(3):268-277.

9. Rosenstock IM, Strecher VJ, Becker MH. Social learning theory and the Health Belief Model. Health education quarterly. 1988;15(2):175-183.

10. Fisher JD, Fisher WA. Changing AIDS-risk behavior. Psychological bulletin. 1992;111(3):455-474.

11. Fisher JD, Fisher WA, Misovich SJ, Kimble DL, Malloy TE. Changing AIDS risk behavior: effects of an intervention emphasizing AIDS risk reduction information, motivation, and behavioral skills in a college student population. Health psychology : official journal of the Division of Health Psychology, American Psychological Association. 1996;15(2):114123.

12. Fisher JD, Fisher WA, Williams SS, Malloy TE. Empirical tests of an information-motivation-behavioral skills model of AIDS-preventive behavior with gay men and heterosexual university students. Health psychology : official journal of the Division of Health Psychology, American Psychological Association. 1994; 13(3):238-250.
13. Bandura A. Health promotion by social cognitive means. Health education \& behavior : the official publication of the Society for Public Health Education. 2004;31(2): 143-164.

14. Bandura A. Human agency in social cognitive theory. The American psychologist. 1989;44(9):1175-1184.

15. Fishbein M. The role of theory in HIV prevention. AIDS care. 2000; 12(3):273-278.

16. Fishbein M. A theory of reasoned action: some applications and implications. Nebraska Symposium on Motivation. Nebraska Symposium on Motivation. 1980;27:65-116.

17. Fishbein M. A reasoned action approach to health promotion. Medical decision making : an international journal of the Society for Medical Decision Making. 2008;28(6):834-844.

18. Albarracin D, Johnson BT, Fishbein M, Muellerleile PA. Theories of reasoned action and planned behavior as models of condom use: a metaanalysis. Psychological bulletin. 2001;127(1):142-161.

19. Rippetoe PA, Rogers RW. Effects of components of protection-motivation theory on adaptive and maladaptive coping with a health threat. Journal of personality and social psychology. 1987;52(3):596-604.

20. Prochaska JO, Velicer WF, Rossi JS, et al. Stages of change and decisional balance for 12 problem behaviors. Health psychology : official journal of the Division of Health Psychology, American Psychological Association. 1994;13(1):39-46.

21. Prochaska JO, Redding CA, Harlow LL, Rossi JS, Velicer WF. The transtheoretical model of change and HIV prevention: a review. Health education quarterly. 1994;21(4):471-486.

22. Rosenstock I, Strecher V, Becker M. Social learning theory and the Health Belief Model. Health Education Quarterly. 1988;15(2):175-183.

23. Fisher JD, Fisher WA, Amico KR, Harman JJ. An informationmotivation-behavioral skills model of adherence to antiretroviral therapy. Health psychology : official journal of the Division of Health Psychology, American Psychological Association. 2006;25(4):462-473.

24. Rivet Amico K. A situated-Information Motivation Behavioral Skills Model of Care Initiation and Maintenance (sIMB-CIM): an IMB model based approach to understanding and intervening in engagement in care for chronic medical conditions. Journal of health psychology. 2011;16(7):1071-1081.

25. Bandura A. Social cognitive theory: an agentic perspective. Annual review of psychology. 2001;52:1-26.

26. Reynolds NR. The problem of antiretroviral adherence: a self-regulatory model for intervention. AIDS care. 2003;15(1):117-124.

27. Barnes AS. Roadmap for implementing the Chronic Care Model for weight management: Healthcare Institution's Response to Obesity (HIRO). Texas Medical Center Dissertations (via ProQuest). AAI1462681. (2009). http://digitalcommons.library.tmc.edu/dissertations / AAI 1462681

28. Wagner EH. Chronic disease management: what will it take to improve care for chronic illness? Effective clinical practice : ECP. 1998;1(1):2-4.

29. Ryan P, Sawin KJ. The Individual and Family Self-management Theory: Background and Perspectives on Context, Process, and Outcomes. Nursing outlook. 2009;57(4):217-225.e216.

30. Andersen RM. Revisiting the behavioral model and access to medical care: does it matter? Journal of health and social behavior. 1995;36(1):110.

31. Gelberg L, Andersen RM, Leake BD. The Behavioral Model for Vulnerable Populations: application to medical care use and outcomes for homeless people. Health services research. 2000;34(6):1273-1302.

32. Busza J, Walker D, Hairston A, et al. Community-based approaches for prevention of mother to child transmission in resource-poor settings: a social ecological review. Journal of the International AIDS Society. 2012;15(Suppl 2) 17373.

33. Bronfenbrenner U. Making Human Beings Human: Bioecological Perspectives on Human Development:. Thousand Oaks: Sage Publications. 2005.

34. Conn VS, Enriquez M, Ruppar TM, Chan KC. Meta-analyses of Theory Use in Medication Adherence Intervention Research. American journal of health behavior. 2016;40(2):155-171.

35. Miller WR, Rollnick S. Motivational Interviewing: Helping People Change: Guilford Press; 2012.

36. Miller WR, Rose GS. Toward a theory of motivational interviewing. The American psychologist. 2009;64(6):527-537.

37. Webb TL, Joseph J, Yardley $\mathbf{L}$, Michie S. Using the internet to promote health behavior change: a systematic review and meta-analysis of the impact of theoretical basis, use of behavior change techniques, and 
mode of delivery on efficacy. Journal of medical Internet research. 2010;12(1):e4.

38. Conn VS, Ruppar TM, Chase JA, Enriquez M, Cooper PS Interventions to Improve Medication Adherence in Hypertensive Patients: Systematic Review and Meta-analysis. Current hypertension reports. 2015; 17(12):94.

39. Amico KR, Harman JJ, Johnson BT. Efficacy of antiretroviral therapy adherence interventions: a research synthesis of trials, 1996 to 2004 Journal of acquired immune deficiency syndromes. 2006;41(3):285-297.
40. Tague NR. The Quality Toolbox, Second Edition: ASQ Quality Press; 2005.

41. Glanz K, Rimer BK, Lewis FM. Health Behavior and Health Education: Theory, Research, and Practice: Jossey-Bass; 2002.

42. Wight $\mathbf{D}$, Wimbush $\mathbf{E}$, Jepson $\mathbf{R}$, Doi $\mathbf{L}$. Six steps in quality intervention development (6SQUID). Journal of epidemiology and community health. 2016;70(5):520-525.

43. Bartholomew LK, Parcel GS, Kok G. Intervention mapping: a process for developing theory- and evidence-based health education programs. Health education \& behavior. 1998;25(5):545-563. 